\title{
LOS ÚLTIMOS SIETE DÍAS DE LA VIDA DE K. LA CATEGORÍA DE TIEMPO EN EL CASTILLO DE FRANZ KAFKA
}

Luis A. Acosta

Hablar de la categoría de tiempo en crítica literaria es una cuestión que plantea no pocas dificultades, de una manera especial si se tienen en consideración la acepción o las distintas acepciones que la crítica de los últimos decenios, especialmente el estructuralismo, ha otorgado al concepto.

A la hora de precisar el concepto cabe la posibilidad de dejarse enseguida llevar por las acepciones más clásicas confirmadas por la filosofía. No sería, en último término, incorrecto si se parte, como aquí se hace, del reconocimiento de la realidad del sistema literario, en la que los factores de naturaleza cultural, filosófica etc., cobran una relevancia necesaria a la hora de entender el fenómeno literario, y no sólo como factor externo a la obra, sino como factor constitutivo de la misma.

Dejarse llevar por la consideración del concepto de tiempo propio de la filosofía clásica, significa entenderlo como una categoría objetiva tal y como formularan Platón y Aristóteles. Se trata de una serie sucesiva de puntos temporales dentro de la que tiene lugar cada uno de los estados puntuales del acontecer de la realidad. Por otro lado, una realidad temporal de esta categoría, es decir, mensurable tiene mucho que ver a la hora de su comprensión con la realidad espacial; significa igualmente entenderlo de esta manera, cierto que desde las variantes introducidas por científicos como Leibniz o Newton referidas al tiempo objetivo como realidad absoluta, o la introducida por Kant en cuanto realidad empírica o idealidad transcendental que no dispone de existencia por sí misma, sino sólo en cuanto forma configurada apriorísticamente por el sujeto.

Desde estas perspectivas, de prácticamente única fundamentación, pero de variantes matizadoras y como apoyatura para determinar un concepto específico de literatura, podemos fácilmente explicarnos la teoría literaria de Aristóteles en lo que se refiere al tiempo y la aceptación de su validez hasta los movimientos clásicos y clasicistas e incluso hasta el siglo XIX. Podemos perfectamente explicarnos la exigencia de la unidad de tiempo en la configuración del género dramático, de igual manera que de la práctica de la presentación literaria de la novela y en general de toda la narrativa tradicional, en cuanto manera de presentación sucesiva de los acontecimientos narrados y novelados. En ella el discurso narrativo no sufre grandes alteraciones con respecto a lo que la concepción del autor o del lector está acostumbrado según los principios que caracterizan una concepción empírica del discurso temporal. 
Ahora bien, esta concepción, permítase denominar, clásica del tiempo, en un momento determinado de la historia sufre una alteración muy significativa y, aunque es algo que se nota en todas las manifestaciones que son producto de la actividad intelectual humana, especialmente la artística, es, una vez más, la filosofía la disciplina que se ocupará de encontrar la formulación adecuada. Y será el filósofo francés de la filosofía de la vida (= Lebensphilosophie) Bergson (1859-1941), emparentado con Dilthey y Nietzsche, quien establece los fundamentos para una nueva concepción del tiempo que, considerada desde la evolución del pensamiento filosófico, puede entenderse ex negativo como la oposición a esa concepción clásica racionalista y mecanicista. En este sentido el tiempo se entiende como una sucesión externa a la conciencia humana, y por tanto, como hemos mencionado, medible, pero como aportación intelectual filosófica en sí, en cuanto concepto interno y auténtico, el tiempo del puro movimiento del fluir o de la duración. Para Bergson la duración (= durée) se descubre en el "fluir de la vida interior". "Toda duración auténtica es la forma que toma la sucesión de los acontecimientos de nuestra conciencia cuando nuestro yo se entrega a la vida, cuando se prescinde de llevar a cabo una separación entre los estados actuales y anteriores"1.

Husserl (1859-1938) nacido el mismo año que Bergson, precisará la nueva concepción estableciendo una diferencia fenomenológica entre el tiempo cósmico cuantificable y el tiempo de la conciencia interna de las vivencias en cuanto forma unitaria de todas ellas dentro de la corriente vivencial general. A ello Heidegger (1889-1976), por su parte, añade la peculiaridad de que el tiempo es una realidad que se constituye como el horizonte de toda comprensión del ser, la temporalidad (presente, pasado, futuro) como estructura básica de la existencia humana; una existencia en la que el futuro se hace posible a partir de la existencia del ser humano como hombre y sobre el que se hacen posibles presente y pasado. Se ha dicho que Heidegger en la filosofía, al igual que Picasso en la pintura y Kafka en la literatura, revolucionaron intelectualmente el siglo $\mathrm{XX}^{2}$.

Pues bien, llevadas estas reflexiones al campo de la literatura como premisas necesarias para la comprensión de manifestaciones concretas de la misma, nos encontramos con la simple reflexión siguiente: si el pensamiento filosófico forma parte del sistema literario, el pensamiento filosófico en lo que a la concepción del tiempo se refiere, puede ofrecer el paradigma adecuado, el modelo de fundamentación correcto para la comprensión del fenómeno concreto. Es a todas luces lógico que nos encontraremos ante un camino errado si para entender una obra clásica en el sentido que de época tiene este término, nos servimos del procedimiento fundamentado en una concepción del tiempo propio de la filosofía de la vida o propio de la filosofía existencial. De igual manera nos resultará imposible llegar a conclusiones definitorias sobre las obras de autores no clásicos - otra vez en el sentido de época -, si hacemos uso de concepciones del tiempo propias de la filosofía aristotélica en su

\footnotetext{
${ }^{1}$ Según Martin Weinmann en Metzler Philosophen Lexikon. Von den Vorsakratikern bis zu den Neuen Philosophen. Unter Redaktionmitarbeit von Christel Dehlinger, Klaus Fischer, Thomas Jung und Heidi Oßmann. ed. de Benrd Lutz. Stuttgart: J.B. Metzlersche Verlagsbuchhandlung, págs. 98-99. En su primera obra Essai sur les données immédiates de la conscience, 1889. (Dt. 1911, Zeit und Freiheit. Eine Abhandlung über die unmittelbaren Bebußtseinstatsachen)
}

${ }^{2}$ Según Silvio Vietta, ibid. pág. 326. 
más amplio sentido de la palabra. Todo ello naturalmente desde esta consideración filosófica del tiempo tamizada por los conceptos respectivos propios de la crítica literaria.

La validación de este argumento puede encontrar su lugar en el análisis e interpretación de la configuración que del tiempo se lleva a cabo en la obra de Kafka El castillo.

Si nos atenemos a la concepción del tiempo como un fluir cuantificable, es decir, aristotélico, a lo largo de la obra nos encontramos con datos más que suficientes como para realizar una operación de este tipo. Desde la observación del tiempo objeto de la narración de los acontecimientos novelescos, según manifestación de $\mathrm{K}$. en el último capítulo de la obra, en que al final ya del mismo la posadera le enseña la ropa que tiene acumulada en los armarios, los acontecimientos se extienden, digamos a una semana, Sie sind mir aufgefallen, gleich als ich dich das erstemal sah, es war vor einer Woche etwa, hier, im Flur $^{3}$. Pero independientemente de este dato, que por otra parte no es exacto en su expresión, a lo largo de la obra hay referencias más que suficientes que permiten concluir que se trata en efecto de un tiempo que se extiende justamente a una semana.

El problema que se plantea en esta obra, podría abordarse analíticamente teniendo en consideración conceptos propios más de la crítica literaria que de la filosofía. Porque independientemente de que, como acabamos de ver, se trate de un tiempo de una semana por el que se extiende la historia de la novela, a todos se nos antoja que no se pueden incluir, aunque sea una novela de quinientas páginas, como es el caso, todos los acontecimientos que pueden protagonizar los personajes de la misma dentro de una semana, de la misma manera que, por otra parte, una semana de tiempo puede, en principio, parecer insuficiente para llenar una obra novelesca de esa extensión. Pero, hay una prueba evidente, y es que en El castillo, sin corresponder ni a lo uno ni a lo otro, Kafka consigue, y ya lo creo que lo consigue, una novela de esa extensión, en la que, podría afirmarse que, independientemente de que sea fragmentaria, no le falta ninguno de los ingredientes que permiten considerarla, no sólo una gran novela, sino una de las más grandes del siglo XX.

Para explicárnoslo necesitamos de otros criterios. De entrada, tenemos que olvidarnos del tiempo cronológico cuantificable de la experiencia de todos los días y tener además muy presente que el tiempo de la novela, sea más próximo o sea más alejado del tiempo astrológico, es un tiempo de características completamente diferentes y peculiares que, en muchas ocasiones y sobre todo en la novela moderna, no tienen mucho que ver entre sí.

En primer lugar partimos del hecho de que la realidad de la obra literaria en general y de la novela en particular es una realidad que se entiende como pseudorreferencial. Es decir, como algo que, si bien tiene que ver con un mundo que la transciende y del que incluso toma su fundamento, no es, a fin de cuentas, más que una realidad que hace referencia al mismo de una manera sólo aparente. Porque lo que de hecho ocurre, es que el mundo de ficción resultante es un mundo que está estructurado única y exclusivamente desde los fundamentos de la fícción literaria. Y estos fundamentos sólo se encuentran dentro de la totalidad que constituye la obra, se explican dentro de la misma y en relación con todos los 
que la estructuran, y es en ella donde cobran su naturaleza específica. Con lo que esa referencialidad aparente o pseudorreferencialidad podría expresarse mejor con el término autorreferencialidad, y es de este modo como inicialmente puede entenderse la estructura de la obra novelesca desde y dentro de la totalidad que la constituye.

Uno de los muchos elementos que la integran es, como no podía ser de otra manera, el tiempo, y ello con las múltiples formas de tratamiento del mismo que conlleva, en el que, sobre todo en la novela moderna, la linealidad es algo que destaca por su ausencia.

Porque el tiempo de la ficción es un tiempo que sólo se manifiesta como un fluir del acontecer narrativo organizado únicamente por la conciencia del autor, en un primer lugar, pero organizado por la conciencia del lector en el momento definitivo en que se constituye la obra literaria. Porque independientemente de como organizara Kafka en su conciencia de escritor en el acto de creación literaria la obra en cuestión, es el lector el que en definitiva va a dar forma en su conciencia a la totalidad de la obra a partir de las orientaciones que ofrece el texto.

Cuando el lector desarrolla la tarea de leer una obra, está activando la conciencia imaginativa personal a partir de los datos aportados por el texto, y lo hace constituyendo una realidad mental que cobra visos de desarrollo protencional, es decir, en permanente movimiento hacia adelante, en protención, digamos, hacia el tiempo futuro. La realidad que se va constituyendo permanece anclada en la conciencia del lector, lugar donde va activándose a medida que se reciben nuevos datos. Con lo que el proceso de protención, o de desarrollo hacia el futuro, se complementa con el de retención, el de tendencia hacia atrás, hacia la parte de la realidad ficticia narrativa que ya se ha establecido y que, mediante los nuevos datos que se reciben constantemente, la realidad ya constituida parcialmente puede ser sometida a un proceso de verificación y en consecuencia también de cambio.

Uno de los elementos que colaboran de una manera especial en este proceso es precisamente el elemento temporal. Por tanto, lo que realmente interesa en el caso concreto es el análisis de la forma específica que toma ese tiempo en las conciencias respectivas y que la crítica entiende como tiempo narrado (dt. erzählte Zeit, engl. story time = tiempo de la obra - frente a Erzählzeit / discourse time = tiempo indirecto, tiempo de lectura y de escritura). Ahora bien, hay que tener en consideración que ese tiempo de la narración se manifiesta normalmente en un grado de mayor o menor concentración para de este modo hacer posible la narración de hechos y acontecimientos, lo que sería imposible de presentar desde las condiciones propias del tiempo astronómico cuantificable.

Ahora bien, dentro de este discurso argumental teórico, lo importante radica en el hecho de que el tratamiento específico que el autor otorga al tiempo, es decir, el tiempo narrado, estos es, las formas de manejo del mismo, como pueden ser las de concentración, de prolepsis o analepsis etc., aportan un punto de referencia fundamental en el acto de recepción como tal, y en el de recepción interpretativa en particular.

Desde esta perspectiva nos encontramos en mejor disposición de entender la forma cómo en El castillo el autor se sirve del tiempo. Para ello, y nada más que como punto de referencia, nos servimos de las alusiones concretas que en la obra se hacen respecto del 
tiempo, que nos lleva a unos resultados que, inicialmente, podrían dar la impresión de que en la novela lo que se ofrece es una secuencia temporal claramente delimitable.

Observamos que en un momento determinado, es por la tarde, el agrimensor K. llega a un pueblo camino del castillo y en una posada, Posada del Puente, busca alojamiento. A la mañana siguiente se pone en dirección del castillo al que no llega, después de lo cual termina en otra posada, esta vez en la Posada señorial donde pasará la segunda noche. El tercer día lo pasa durmiendo. La tercera mañana, es decir, el cuarto día, se entrevista con el alcalde de la localidad y pasa la noche en la casa de la escuela de la localidad. El día siguiente es un día de intensa actividad en el que ocurren una serie amplia de acontecimientos. Pasará la noche de nuevo en el la Posada señorial. En la mañana del sexto día será despertado por la camarera de la posada que le invita a vivir con él. Aquí se interrumpe la novela que, como fragmentaria que es y según testimonio del amigo de toda su vida Max Brod, el último día del plan de la novela estaba previsto para el de la muerte del protagonista a quien le faltan ya las fuerzas para poder seguir viviendo.

Nadie duda de que una semana es tiempo más que suficiente, aunque tampoco excesivo para llenar la trama de una novela, si bien es cierto que si pensamos en cualquier ejemplo de novela tradicional, en la mayoría de los casos nos vamos a encontrar con fábulas novelescas cuyos acontecimientos se extienden a un tiempo de duración mucho más prolongada. A pesar de ello, en El castillo hay un aspecto que llama enormemente la atención, y es que desde una observación todavía dentro del terreno de lo cuantificable y cuantificable precisamente a través de la medida que supone la cantidad de páginas dedicada a cada uno de los días a que se acaba de hacer mención, nos encontramos con lo siguiente: si el primer día ocupa aproximadamente las 6 páginas del primer capítulo, el segundo ${ }^{4}$ se extiende a lo largo de 50 páginas, resto del capítulo primero y los capítulos segundo y tercero completos, el tercero, ocupa tan sólo una frase del final del capítulo tercero para decir que $\mathrm{K}$. se quedó durmiendo todo el día y toda la noche, el cuarto abarca 108 páginas, es decir los capítulos del cuarto al once incluido, el quinto, el que más extensión ocupa de todos los días, es decir, 205 páginas, desde el capítulo doce hasta el diecinueve, y por fin, el sexto día, las 36 páginas del capítulo veinte y último.

A partir de esta comprobación en la distribución del tiempo, se observa de manera evidente que nos encontramos ante una desproporción manifiesta. Dicho sea de paso, esto no implica, ni muchisimo menos, una afirmación que pueda entenderse con connotaciones negativas en ningún sentido. Porque el tiempo aquí parece tener otro sentido, el tiempo se entiende de otra manera, parece tener otra razón de ser, tener una función que nada tiene que ver con la de medición o cronología. Ya el tiempo astronómico, que de alguna manera podía orientarnos en este sentido por la novela, llama especialmente la atención. En principio, la forma como es presentado literariamente podría muy bien encajar dentro de los parámetros lógicos de una semana de cualquier época del año, pero la atención del lector resulta provocada cuando se observa que, aparte de ser una semana de invierno, las escasas ocasiones en que la acción novelesca, acontecimientos o acciones de los distintos

${ }^{4}$ Comprobar lo que dice Ingeborg Schol en Franz Kafka Der Proze $\beta$ Das Schloß, Interpretation und unterrichtspraktische Vorschläge. Hollfeld, Beyer Verlag, 1980, pág. 67: Er läßt K. außerdem am zweiten Tag seines Aufenthalts feststellen, daß wenn nun schon den vierten Tag hier ist. 
personajes aparecen dentro del marco de la referencia del tiempo astronómico, se trata de un tiempo atmosférico propio del invierno de cualquier país centroeuropeo; un día en el que lo característico del mismo es que siempre está nevando o al menos todo siempre está nevado, un día en el que lo peculiar es que siempre es la falta de claridad diurna, que dura tan poco que parece confundirse con la noche; la noche, por lo demás, nunca es serena, sino más bien turbia y tenebrosa. Cuando, por ejemplo K. llega al pueblo, por cierto carente de nombre alguno, el pueblo, así lo expresa el narrador, está hundido en la nieve. Cuando por alguna circunstancia, lo que ocurre raras veces, $\mathrm{K}$. tiene que salir a la calle, o bien está nevando o bien ha nevado tanto que al andar se hunde necesariamente en la nieve, de manera que el hecho de tener que sacar en todo momento los pies hundidos de la nieve, le produce un cansancio tal que llega hasta necesitar de la ayuda de otros para continuar el camino. Si la salida es por la noche, es entonces una noche tétrica y completamente oscura y la manera de identificación de las personas con las que se encuentra al llegar a la posada, es la de levantar un farol y alumbrar los rostros. Cuando en la obra aparecen - por cierto solamente en una ocasión a lo largo de toda ella - otras estaciones del año, la razón radica, no en la necesidad de las mismas para el desarrollo del discurso novelado. Son simplemente meros puntos situados en el recuerdo, cuya realidad no toma otra entidad que la propia de las realidades del recuerdo y, en consecuencia, más breve que la estación que llena el discurso narrativo.

Pero se hace necesario profundizar todavía más para comprender la configuración que en $E l$ castillo se hace del tiempo si queremos entenderlo de una manera adecuada. A ello ayudará seguramente la observación de algunos ejemplos del llenado novelesco, como son ciertas acciones que se dan en la obra y algunos personajes integrantes de la misma. A partir de ahí es posible cuantificar la realidad del binomio que ellos constituyen en cuanto elementos estructurales propios de la narración.

Como ya se ha mencionado, los tres primeros días del tiempo narrado le hacen pensar al lector, a menos así parece, que se trata de una forma temporal de presentar los acontecimientos similar a como lo hace cualquier novela tradicional; la forma como se llena, como se configura el tiempo en esos días encaja perfectamente dentro de los límites de cualquier día de la vida de una persona no sólo de la novela sino incluso de la vida normal. Otra cosa, naturalmente, son las circunstancias como se produce.

Pero es que aún así, y aquí entran en juego esas circunstancias, en esos primeros capítulos de la obra nos encontramos ante una serie de acontecimientos que parece que van a constituir la razón de que a la vez que se hace necesario esclarecerlos, son los que hacen que el tiempo se llene de tal manera que lleva a una concentración más allá de los límites imaginables, a que el tiempo no sólo se detenga, sino que incluso llegue a desaparecer. Y ello hasta el punto de que al lector llega a resultarle profundamente irritante algo que no sólo está obligado a aceptar, sino que hasta se encuentra en la situación de tener que aceptarlo sin más.

Pero veamos algunos detalles. En esos tres primeros días que ocupan tres capítulos de la obra, que en realidad no son más que dos, puesto que, como hemos visto, el tercero, el protagonista $\mathrm{K}$. se lo pasa durmiendo, en esos tres días se establecen los presupuestos necesarios para el proceso protencional mediante el que el lector va constituyendo el planteamiento y primer desarrollo de la totalidad narrativa. Estos presupuestos, conviene 
insistir, parecen encajar perfectamente dentro de un planteamiento novelesco que da la impresión inicial de que va a respetar un desarrollo normal, y por ello cuantificable, del elemento estructural temporal de la misma.

La novela empieza con la narración del tiempo de la llegada de $\mathbb{K}$. a un pequeño pueblo muy cerca del cual está el castillo, situado en una colina rodeada de niebla y oscuridad, donde viene a trabajar contratado como agrimensor. Como es por la tarde y es una tarde de invierno, lo primero que hace es buscar alojamiento en una posada. A pesar de que surge una dificultad, consistente en que en territorio condal no se puede permanecer sin el correspondiente permiso, y K. no dispone del mismo, sin embargo, mediante una llamada al castillo el asunto se soluciona y $\mathrm{K}$. puede quedarse a dormir (Capítulo I). Al día siguiente, después de desayunar, se pone en marcha en dirección del castillo. En el camino se encuentra con un grupo de niños a quienes acompaña el maestro del pueblo. Pleno de cansancio entra en la casa del curtidor Lasemann. Avanzada la tarde vuelve a la posada. (Capítulo I, final) Se encuentra con los ayudantes que le han sido asignados, y con ellos hace los planes para el trabajo del día siguiente (el tercer día). Mientras tanto le llega un mensajero, Barnabás, con una carta del castillo, en la que se le comunica que ha sido contratado como agrimensor y en la que se habla de las condiciones de trabajo, en ella se dice que él - Barnabás - será el contacto con el jefe y que su inmediato superior será el alcalde del pueblo. Conocerá a la familia de Barnabás, a sus padres enfermos y a sus dos hermanas Olga y Amalia. Rechaza el ofrecimiento de quedarse a dormir en casa de ellos y busca alojamiento, acompañado por Olga, en la Posada señorial donde esa noche se aloja Klamm el jefe de personal del castillo (Capítulo II, segundo día). Al comienzo del capítulo III K. conoce a Frieda, la camarera de la Posada señorial, con quien $\mathrm{K}$. entra en contacto y allí pasan la noche juntos. A la mañana siguiente se levantan, se van a la otra posada, la Posada del puente, donde $\mathrm{K}$. se pasa todo el día durmiendo. Al despertar comienza ya el cuarto día de tiempo narrado que es a su vez también el cuarto de estancia en el pueblo.

Como ya se ha mencionado, nos encontramos con un tratamiento del tiempo perfectamente cuantificable y un llenado del mismo a través del que se produce con maestría narrativa especial la presentación de una acción novelesca a través de los elementos desencadenantes que la hacen posible, una organización de la misma desde la lógica más palmaria. El tratamiento del tiempo no resulta de ninguna manera especialmente llamativo. Sin embargo, un tiempo narrado perfectamente observable y que al lector le ofrece las premisas necesarias, ajenas a cualquier complicación, para a partir de ellas poder ir constituyendo en su conciencia en acto de protención la realidad ficticia de la novela, le dará pie al mismo tiempo en repetidas ocasiones a momentos de incertidumbre en su actividad. En esos primeros días van a emerger aspectos y elementos que al lector le suponen una serie de espacios vacíos que necesariamente tiene que ir llenando, aunque sea, claro está, de manera provisional, lo que puede que más tarde o bien confirme o bien tenga que reorientar. Veamos una vez más. En la noche de la llegada de K. a la posada, independientemente de que necesitara un permiso para permanecer en territorio condal, sorprende el hecho de que mediante la correspondiente llamada telefónica pueda verse que en el castillo no se tenga noticia alguna sobre un agrimensor. Cuando $\mathrm{K}$. se encuentra con el maestro en su intento de primera llegada al castillo, en un momento determinado, el maestro, sin razón de fondo aparente, y la ocasional de que los niños no lo entiendan, comienza a hablar en francés. Y en la continuación hacia el castillo, va por un camino que 
discurre por una serie de curvas y vericuetos que en vez de aproximarlè a su meta, lo que hace es alejarle: “... Y así se puso de nuevo en marcha y prosiguió su camino, pero fue un largo camino. Pues la carretera, esa calle principal del pueblo, no llevaba a la colina del castillo, sino tan sólo a las proximidades del mismo, pero luego, como a propósito, se desviaba, y si bien no se alejaba del castillo, tampoco se aproximaba a él. K. siguió esperando a que, por fin, la carretera se desviara necesariamente en dirección del castillo y sólo por que eso era lo que esperaba, continuó caminando;..." Cuando en el primer día de estancia de K. en el pueblo, se ha hecho de noche ya, y quiere continuar su camino, el carretero Gestäcker se ofrece a llevarle. No obstante, cuando sabe que el destino es el castillo, sin dar explicación alguna, dice que allí no quiere llevarle. En la posada hace saber que al día siguiente llegarán sus ayudantes con los aparatos de agrimensura. Sin embargo, el segundo día se encuentra con unos ayudantes que han sido puestos a su disposición que no entienden absolutamente nada de agrimensura. Con ellos, no obstante, como hemos visto, fija los planes necesarios para el comienzo del trabajo al día siguiente. Cuando para poder acercarse al castillo se pide por teléfono el correspondiente permiso para los ayudantes, el funcionario de turno le dice ni más ni menos a uno de los ayudantes que no se les concederá permiso ni para mañana ni para nunca. Puesto $K$. al teléfono, no recibe otra respuesta que la dada a aquéllos. Pero con un agravante: cuando $\mathrm{K}$. se pone al teléfono, no se identifica como el agrimensor, sino como uno de los ayudantes, haciéndolo mediante un juego que llama poderosamente la atención: ... "K. no hizo caso de esta observación y dijo con repentina decisión: "Al habla el ayudante del señor agrimensor." ¿Qué ayudante? ¿Qué señor? ¿Qué agrimensor?" A K. le vino a la memoria la llamada del día anterior. "Pregunte usted a Fritz" dijo escuetamente. Para sorpresa suya surtió efecto. Pero más que del hecho de que surtiera efecto, se sorprendió de la uniformidad del servicio. La respuesta fue: "Ya lo sé. El eterno agrimensor. Sí, sí. ¿Qué más? ¿Qué ayudantes?" "Josef”, dijo K. Le molestaba un poco el murmullo que hacían los campesinos a sus espaldas; no estaban al parecer de acuerdo con que no se diera a conocer de forma correcta. Pero K. no tenía tiempo para ocuparse de ellos, en ese momento le absorbía la conversación. “¿Josef?” replicó el otro. "Los ayudantes se llaman - una pequeña pausa, era claro que requería que alguien le dijese los nombres - "Artur y Jeremias." "Estos son los nuevos ayudantes", dijo K. "No, son los viejos." "Son los nuevos, y yo soy el viejo que ha llegado hoy tras el señor agrimensor." “No!”, se oyó gritar. “¿Quién soy yo entonces?” preguntó K., tranquilo como hasta el momento. Y después de una pausa dijo la misma voz con el mismo defecto en el habla, pero que era como otra voz más profunda, más respetuosa: "Eres el viejo ayudante". ${ }^{6}$

5 ... So ging er wieder vorwärts, aber es war ein langer Weg. Di Straße nämlich, diese Hauptstraße des Dorfes, führte nicht um Schloßberg, sie führte nur nahe heran, dann aber, wie absichtlich, bog sie ab, und wenn sie sich auch vom Schloß nicht entfernte, so kam sie ihm doch auch nicht näher. Immer erwartete K., daß nun endlich die Straße zum Schloß einlenken müsse und nur, weil er es erwartete, ging er weiter;... op, cit. págs. 20-21

${ }^{6} \mathrm{~K}$. ging auf diese Bemerkung nicht ein und meldete mit einem plötzlichen Entschluß: "Hier der Gehilfe des Herrn Landvermessers." "Welcher Gehilfe? Welcher Herr? Welcher Landvermesser? K. fiel das gestrige Telephongespräch ein. "Fragen Sie Fritz", sagte er kurz. Es half, zu seinen eigenen Erstaunen. Aber mehr noch als darüber, daß es half, staunte er über die Einheitlichkeit des Dienstes dort. Die Antwort war: "Ich weiß schon. Der ewige Landvermesser. Ja. Ja. Was weiter? Welcher Gehilfe?" "Josef”, sagte K. Ein enig störte ihn hinter seinem Rücken das Murmeln der Bauern; offenbar waren sie nicht damit einverstanden, daß er sich nicht richtig meldete. K. hatte aber keine Zeit, sich mit ihnen zu bescháftigen, denn das Gespräch nahm ihn sehr in Anspruch. 
K. concentró su oído en la sonoridad de la voz, pasando casi por alto la pregunta: “¿Qué es lo que quieres?" Colgar el auricular era lo que más le habría gustado. De esta conversación ya no esperaba nada. Sólo como obligado preguntó apresuradamente: “¿Cuándo puede mi señor ir al castillo?” "Nunca", fue la respuesta. "Bien”, dijo K. y colgó ${ }^{7}$. En la visita a la familia de Barnabás llama la atención que el mensajero está dispuesto, si así lo quiere K., a que sus padres y hermanas se marchen para no estar presentes en la conversación. Lo que, naturalmente, K. no acepta: ... Una vez que tú lo ordenes, se marcharán enseguida todos; podrías también, si es que te agrada, estar en nuestra casa, pasar la noche aquí. ¿No he hecho bien?” K. no supo contestar. Así pues, había sido un malentendido, un vil y vulgar malentendido, y $\mathrm{K}$. se había entregado por completo. Se había dejado embaucar por la chaqueta ceñida, brillante como la seda, de Barnabás, que en este momento se desabrochaba y bajo la que aparecía una camisa basta, de color gris a consecuencia de la suciedad y por todas partes remendada, sobre el vigoroso y anguloso pecho de un criado. ... ${ }^{8}$ Cuando K. entabla conversación con Frieda y le hace saber que es el amante de Klamm, no se le ocurre otra cosa que sugerirle que sea su amante. Acaba de conocerla, no le dan permiso para ir al castillo y pretende quitarle la amante a su jefe, a quien ni siquiera conoce todavía. Es más, cuando Frieda se acuesta con $\mathrm{K}$. debajo del mostrador de la taberna, - entre los charcos formados por los restos de la cerveza escanciada durante todo el día, - sin duda uno de los pasajes más significativos y más magistralmente contados de la obra - burlando de manera cínica la ronda de inspección del posadero, da la impresión de que de ese encuentro ha surgido un amor profundo. Frieda se ha enamorado de $\mathrm{K}$., $\mathrm{K}$. se ha enamorado de Frieda. Todo, hay necesariamente que reiterarlo, coincidiendo con el segundo día y la tercera noche de su estancia en el pueblo.

Creo que no es necesario aportar más detalles para constatar que la tensión está servida y lo está de manera que el lector sufre una ralentización muy acusada en su proceso protencional; lo que hace que el lector mantenga la tensión hacia adelante y surja, me atrevo ya aquí a afirmar, la necesidad de otro tiempo narrado. El lector se encuentra ante un

"Josef?" fragte er zurück. "Die Gehilfen heißen" - eine kleine Pause, offenbar verlangte er die Namen jemandem anderen ab - "Artur und Jeremias." "Das sind die neuen Gehilfen", sagte K. "Nein, da sind die alten." "Es sind die neuen, ich aber bin der alte, der dem Herrn Landvermesser heute nachkam.". Nein!" schrie es nun. "Wer bin ich also?" fragte K, ruhig wie bisher. Und nach einer Pause sagte die gleiche Stimme mit dem gleichen Sprachfehler und war doch wie eine andere tiefere, achtugnswertere Stimme: "Du bist der alte Gehilfe." (ibid. p. 33-34)

${ }^{7}$ K. en Stimmklang nach und überhörte dabei fast die Frage: "Was willst du?" Am liebsten hätte er den Hörer schon weggelegt. Von diesem Gespräch erwartete er nichts mehr.Nur gezwungen fragte er noch schnell: "Wann darf mein Herr ins Schloß kommen?" "Niemals", war die Antwort. "Gut", sagte K. und hing den Hörer an. ibid. pág. 34

8 ... Sie werden sich alle gleich entfernen, wenn es gefiehlst; auch könntest du, wenn es dir bei uns besser gefällt, hier übernachten. "Habe ich nicht recht getan?" K. konnte nicht antworten. Ein Mißverständnis war es also gewesen, ein gemeines, niedriges Mißverständnis, und $K$. hatte sich ihm ganz hingegeben. Hatte sich bezaubern lassen von des Barnabas enger, seidenglänzender Jacke, die dieser jetzt aufknöpfte und unter der ein grobes, grauschmutziges, viel geflicktes Hemd erschien über der mächtigen, kantigen Brust eines Knechtes. ibid. pág. 46 
cúmulo tal de suposiciones sobre hechos y realidades ya existentes, que para poderlas explicar, no puede hacerlo de la manera cronológicamente, digamos, correcta.

Así pues, nos encontramos ante una linealidad del tiempo fácilmente perseguible en los primeros capítulos, un planteamiento de una acción perfectamente organizada y unas expectativas del lector sobre cómo va a continuar el discurso de la acción narrativa.

El momento en que $\mathbf{K}$. y Frieda se encuentran debajo del mostrador se establece el detonante para el desarrollo del núcleo de la acción de la novela; será, por otra parte, la razón de ser de la marcha específica que toman los acontecimientos. Pero de la misma manera que el acontecimiento fundamental inicial ha ocurrido de una manera, diríase que inesperada, las circunstancias y consecuencias que trae consigo son tantas y tan relevantes, que llevan a que la novelación tanto de las primeras como de las segundas exija un tratamiento del tiempo narrado en un grado alto de concentración; un tiempo que como tal parece desaparecer, y desaparecer en el sentido de que, o bien se paraliza en el presente o bien se paraliza retrocediendo hacia el pasado. Cierto que el autor no va a renunciar a una especie de juego - el juego literario a fin de cuentas - de pretender hacer ver que es fiel a un tiempo real cuantificable, pero consciente sin embargo del todo que el tiempo narrado está rompiendo la totalidad de los esquemas posibles de cuantificación. El resultado no es otro que una concentración de acontecimientos narrados cuyo contenido no cabe dentro de medidas normales. De este modo se está rompiendo un punto de referencia en la organización que se considera tradicional en el tratamiento del tiempo de la novela.

Pero volvamos al cuarto día de estancia de $\mathrm{K}$. en el pueblo próximo al castillo, que ocupa desde el capítulo VI hasta el XI ambos incluidos. Al tiempo que hay que solucionar

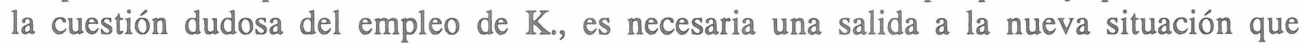
supone la relación entre K. y Frieda. Tanto en lo que se refiere a la primera cuestión, como en lo que se refiere a la segunda, Klamm es la persona de referencia; es, de un lado, la autoridad máxima del castillo, de otro, es además el amante de Frieda. Por otra parte entra en juego la Familia Barnabás, "Esos harapientos infames", como diría la posadera. Se trata de un nuevo elemento en la obra que va a añadir todavía más complicación, un elemento que al lector le supone un factor para cuya comprensión necesita de más datos.

La obra va a estructurarse sobre dos elementos fundamentales: la relación del protagonista con las autoridades, por un lado, y su relación con Frieda. Pero bien entendido que desde el momento en que Frieda ha sido la amante de Klamm, será éste quien sirva de elemento estructural que pone en relación el acontecer de los dos elementos.

El desarrollo novelado del primer elemento encuentra su materialización inicial en la entrevista con el alcalde, entrevista que supone un movimiento temporal hacia atrás. En ella el alcalde del pueblo le hace saber que ya conoce el asunto del agrimensor, pero sabe también que no se necesita un agrimensor, que sí, que hace mucho tiempo, hace años, que llegó un decreto sobre el asunto. Decreto que el alcalde intentará encontrar entre una multiplicidad de legajos, que Kafka describe de una manera magistral, y que no logrará encontrar. 
El tiempo vuelve a detenerse y a moverse hacia atrás cuando Gardena, la posadera le cuenta a K. la historia de hace veinte años de su relación con $\mathrm{Klamm}$. Todo ello cuando es mediodía del cuarto día de la narración.

El tiempo se detiene otra vez en el presente, pero en un presente cada vez más dilatado. Como no se necesita un agrimensor, el maestro del pueblo, en nombre del alcalde, le comunica a $\mathrm{K}$. que se le ha ofrecido el puesto de bedel de la escuela, algo que en realidad tampoco se necesita. K. y Frieda deciden, no obstante, ocupar un aula como vivienda.

Esto ocurre cuando el cuarto día parece ya avanzado. El capítulo VIII comienza narrando que "empezaba realmente a oscurecer..." Pero en ese día van a ocurrir todavía muchas cosas: la presentación de la figura de Pepi, la sucesora de Frieda en el trabajo de la Posada señorial (cap. VIII), la espera de la salida del trineo de Momus el secretario de Klamm a quien ha confundido con éste (cap. VIII), discusión entre Klamm y la posadera sobre la posibilidad de llegar hasta Klamm (cap. IX), la complicación que llega a una situación de las que se conocen como propiamente kafkianas sobre el trabajo, de manera que $\mathbf{K}$. recibe una carta de $\mathbf{K l a m m}$ en la que se dice que está muy satisfecho del trabajo que $\mathrm{K}$. está realizando como agrimensor, lo que resulta incomprensible cuando por la mañana el alcalde le ha dicho que ha sido contratado como bedel. O el tiempo no encaja, o la administración no funciona, o se están burlando de K. o... es simplemente kafkiano.

Con la cena de $\mathrm{K}$. y Frieda y los ayudantes que se les ha asignado, primero para el trabajo como agrimensor y luego como bedel termina el cuarto día. Conviene con todo hacer la observación de que el tiempo de la novela va a extenderse a dos días más y que todavía no ha transcurrido la mitad de la misma. Faltan aún los dos últimos días, los dos días en los que van a ocurrir todavía muchas cosas, los dos días en los que el tiempo sufre el mayor grado de concentración novelesca, por un lado, y de dilatación cronológica, por otra.

Aun así, la mañana del quinto día va a resultar relativamente breve en lo que al tiempo narrado se refiere. Se limita al intento de expulsión de la escuela por parte del maestro y a la expulsión efectiva de los ayudantes por parte de $\mathrm{K}$. Sin embargo será la tarde del quinto día la que va a convertirse en la más rica en lo que a tiempo narrado se refiere y en consecuencia en proliferación de acontecimientos. En ella se confirma el tratamiento del tiempo que se convertirá en paradigmático y original de la novela, si bien hay que hacer la observación que cronológicamente se extenderá a prácticamente toda la noche del sexto día.

Todo comienza con la visita a la familia de Barnabás el mensajero, la familia que, caída en desgracia, se encuentra prácticamente marginada. Aquí el tiempo vuelve a detenerse otra vez, vuelve a moverse hacia atrás y va a simultanear un movimiento de presente como un movimiento hacia atrás, en definitiva en un movimiento circular.

Se detiene con la narración de la relación de Barnabás con el castillo, la consideración y relación de la gente con las mismas, la relación de Barnabás con Klamm en su trabajo para el castillo, si es Klamm para quien realmente trabaja Barnabás, la personalidad de Klamm. Pero el tiempo se mueve hacia atrás con la narración de acontecimientos que explican la situación de aislamiento de la familia y el surgimiento de la complicación de la relación entre Barnabás y Frieda. 
El tiempo se mueve hacia atrás con la narración del secreto de Amalià, que ocurrió hace tres años y que no consistió más que en la negativa de acceder a los deseos de un funcionario del castillo, Sortini, sobre Amalia, la hermana de Barnabás en una fiesta de bomberos. La negativa consagra la desgracia de la familia. Su castigo recae sobre toda la familia, cuyas consecuencias son, además de las mencionadas, las enfermedades de los padres, ya ancianos, aunque la relativamente positiva del trabajo de Barnabás como mensajero, que ha ocurrido hace solamente una semana. Con ello la historia de esos tres años del acontecimiento, las consecuencias a él debidas, y el trabajo de Barnabás hacen mantener la continuidad del presente narrado.

La vuelta a la narración del presente lleva a la situación de que este encuentro en la casa de los Barnabás es la razón de la ruptura de Frieda con $\mathrm{K}$. Pero aquí surge algo realmente llamativo para el lector. Los acontecimientos se precipitan de tal manera que el lector no entiende qué es lo que realmente ha pasado. Advierte que ha ocurrido algo que no ve, que han ocurrido cosas, pero que el tiempo dedicado a ellas ha sido tan llamativamente corto que no parece razón suficiente como para haber llegado a esa situación. De tal manera que el tiempo, a parte de detenerse, no sólo no se mueve hacia atrás, sino que parece tomar consistencia sólo en la mente del lector a partir de los indicios que le ofrece la autoridad autorial. Para K. el problema con las autoridades se ha agravado con el problema de su relación con Frieda. La decisión de separarse no va a encontrar razonamiento ninguno que lo explique.

El detenimiento del tiempo en su configuración como presente llega a su grado extremo, cuando junto a la citación de $\mathrm{K}$. junto a Erlanger, es decir, cuando al problema de la relación con la autoridad, se añade el de la relación con Frieda. La cita es por la noche, Frieda considera que $\mathbb{K}$. ha sido infiel y mientras tanto ha sido contratada otra vez para trabajar en la posada en que ya había trabajado antes. Todo ello lo discuten $\mathrm{K}$. y Frieda en el pasillo de la posada en que se hospeda Erlanger, el sustituto de Klamm; la argumentación de Frieda es que de eso ya han hablado muchas veces. ¿Cuándo, si prácticamente no llevan más que un día juntos? Pero es que además en la espera para ver a Erlanger ocurren todavía muchas cosas más; hasta el punto de que el tiempo cronológico se mueve, incapaz de absorber todos los acontecimientos, hasta avanzada la noche. Son las cinco de la mañana cuando K. logra ver a Erlanger quien le comunica que Frieda tiene que volver a la cantina. La sorpresa para el lector es otra vez llamativa: la comunicación de Erlanger de algo que ya sabe $\mathrm{K}$. recuerda a aquella otra en que se le dijo que estaban contentos con su trabajo de agrimensor cuando en realidad trabajaba ya como bedel. Pero el presente sigue sin progresar. K. sigue en el pasillo contraviniendo la reglamentación que se lo prohibe.

Con todo, K. logra que le permitan dormir en la posada. Es la mañana del sexto día del que va a pasar doce horas durmiendo. Con ello el tiempo real va a limitarse considerablemente. Y es poca la actividad narrativa de acontecimientos de acción. En él, no obstante, parece haber tiempo, sirva la redundancia, para todavía poder explicar algo de lo que ha ocurrido. Para poder explicar, aunque sólo sea por parte de uno de los personajes menos significativos de la cobra, como es Pepi, la sustituta en el trabajo de Frieda durante la ausencia de ésta, pero que ahora tiene que dejar de serlo. Pepi en un diálogo con $\mathrm{K}$. le explica cómo era su vida hasta antes de que él apareciera en el pueblo, cómo Frieda había sido antes que de K. fuera la amante de Klamm; cómo Frieda, ante la invariabilidad de su 
situación decidió entregarse al más humilde de los humildes, decidió entregarse a K., cómo Frieda ha jugado con $\mathrm{K}$. en la persona de uno de los ayudantes, Jeremías, cuando $\mathrm{K}$. está fuera, cómo, en definitiva, Frieda ha engañado a $\mathrm{K}$., y finalmente cuáles son las consecuencias que todo ello tiene para su vida. Pero en este diálogo se aprecia una vez más cómo el tiempo narrado no corresponde con el tiempo real. Pepi hace ver que los cuatro días de trabajo en la cantina de la posada como sucesora de Frieda, han sido cuatro días de los más importantes y prometedores de toda su vida. Independientemente de que ello concuerde o no con la realidad, si nos atenemos al relato, esa medición del tiempo no corresponde con el real, pues de hecho Pepi no ha trabajado más que dos días en la cantina de la posada, lo que en el proceso de recpeción de la realidad literaria por parte del lector constituye una orientación muy significativa para consumar el proceso de actualización creativa de la construcción literaria. Porque si bien es cierto que a lo largo de la obra no se han producido incoherencias cronológicas en el tratamiento del tiempo narrado en su correspondencia con el tiempo cronológico, aquí, precisamente al final de la obra, se produce esta alteración. Y no es que pueda considerarse un lapsus, podríamos decir, narrativo del autor; Pepi, habla en repetidas ocasiones y con insistencia de esos cuatro días de su vida. Naturalmente $\mathrm{K}$. no está de acuerdo con la explicación que ella da sobre la actuación de Frieda. K. cree que Frieda no ve la realidad tal y como es. No obstante, sus intereses por $\mathrm{K}$. hacen que le invite, ahora que lo ha perdido prácticamente todo, a compartir la habitación, pequeña pero acogedora, que ya comparte con otras dos sirvientas. Es la manera de ofrecerle una forma más agradable de sobrellevar el largo invierno, al final del cual parece ofrecérsele la todavía lejana, pero posible llegada de la primavera, lo que habría entenderse como un motivo de esperanza.

En suma, de todo ello se aprecia un movimiento lento en la progresión del tiempo, que se comprueba en la práctica paralización dentro del presente, que cuando da la impresión de que va a llegar al final de una secuencia narrativa, la duración del mismo se dilata, con lo que el tiempo de la narración se configura con mayor amplitud que el tiempo narrado. Dicho de otra manera, la progresión del tiempo está sometida a un proceso de concentración, se configura una secuencia temporal cuyas unidades se conforman en un movimiento de concentración mediante la presentación de una multiplicidad de acontecimientos que no parece tener fin, de una serie de acontecimientos, en cualquier caso, que no tendrían posibilidad de integrarse dentro de un tiempo, digamos real (cap. XV cuando parece que K. se marcha de casa de los Barnabás, pero al final se queda y se queda mucho tiempo, al menos mucho tiempo narrado). Se comprueba, en segundo lugar, mediante el desplazamiento del decurso temporal hacia el pasado, con lo que muchas secuencias de la realidad fabulada, que tienen lugar en el presente, encuentran su explicación en secuencias de la realidad del pasado, que mediante la técnica del diálogo o de la narración simple cobran realidad novelada actual (secreto de Olga, familia Barnabás, pasado de Frieda, de Pepi, de la posadera etc.). Mediante, en tercer lugar, el desplazamiento del tiempo en protención insinuada hacia el futuro, si bien se trata de un procedimiento utilizado con mucha menor frecuencia ( $\mathrm{K}$. quiere hablar con $\mathrm{Klamm}$, la posadera quiere hacerle ver que esto no va a tener lugar tal y cómo de hecho será).

Todo ello lleva a la conclusión ya insinuada anteriormente de que el tiempo narrado nada tiene que ver con el tiempo cronológico; que la alteración de secuencias del presente con secuencias que tienen lugar en el pasado, llevan de hecho a la eliminación de ese 
tiempo, lleva a una construcción temporal tal y como ocurre en la conciencia del autor dentro de la que el presente se simultanea y mezcla con el pasado que narrativamente se hace presente; conciencia del autor en la que tanto presente como pasado hecho presente, crean una proyección protencional hacia un futuro prácticamente inexistente. El protagonista se encuentra en una situación en la que fuera de los deseos de salida del estado existencial absurdo en que se encuentra en el presente, está prácticamente desdotado de la posibilidad de futuro, una circunstancia que el lector advierte de inmediato. Y aunque la obra no termine, el final que sabemos que el autor tenía previsto proporcionarle, no es otro que el de la inmersión y hundimiento en un presente, que como inmóvil que es, no puede ofrecer una salida. De acuerdo con el relato de Max Brod, el amigo de Kafka, el protagonista $\mathbb{K}$., a pesar de que recibiría la autorización para residir en territorio del castillo, el hastío y cansancio del presente inamovible, le llevaría irremediablemente a la muerte.

Podríamos preguntarnos, ¿para qué entonces esos seis días de novela, que de haberse concluido según el proyecto del autor, serían siete? A esta pregunta se puede contestar diciendo que, independientemente del significado que pueda tener desde alguna perspectiva cultural-confesional esa semana de tiempo - no olvidar que Kafka era judío -, el tiempo narrado de esos días no ha de ser entendido más que como un recurso narrativo, un recurso literario que, junto con otros más, constituyen el código genérico específico, la gramática literaria que establece la estructura narrativa de la obra dentro de la que la fábula cobra su propia configuración. Son siete días de la vida de $\mathbb{K}$. en los que todo lo que ocurre es razón suficiente para que el protagonista se prepare para la meta existencial que es la muerte, aunque, como diría Pepi, haya una lucecita, aunque la llegada de la primavera pueda ofrecer ciertos visos de esperanza al final del túnel. Esta ambivalencia queda perfectamente fijada con una específica utilización del tiempo. 